\title{
EXCHANGE STIFFNESS PARAMETER AND MAGNETIZATION IN PSEUDOPOTENTIAL CALCULATIONS
}

\author{
W. SCHMIDT \\ Institute of Molecular Physics, Polish Academy of Sciences \\ Smoluchowskiego 17, 60-179 Poznań, Poland
}

\begin{abstract}
We study low temperature magnetic properties of transition metals. The ground state spontaneous magnetization is calculated in a pseudopotential approximation. In the calculations, the core electronic orbitals are approximated by asymptotic expressions. The magnetizations are calculated for uniform spin densities of valence (chemically active) electrons and in the Lindhard approximation. Our calculations of the Bloch wall parameter and the magnon stiffness constant are based on a study of ferromagnetic and paramagnetic phases. A simple Bragg-Williams approximation between energy of spin polarization and the time-average of the spin moment per atom determines the "exchange coupling" (Heisenberg-like) for spin-polarized nearest neighbour Wigner-Seitz cells. $A b$ initio numerical results of the spontaneous magnetization, the Bloch wall parameter, and the magnon stiffness constant are obtained for $\mathrm{Fe}, \mathrm{Co}$, and $\mathrm{Ni}$.
\end{abstract}

PACS numbers: 71.15.Mb, 71.15.Hx, 75.30.Et

\section{Spontaneous magnetization in a pseudopotential calculation}

The density functional theory (DFT) $[1,2]$ recast the problem of the many-electron wave function in terms of the electronic density (or the spin densities) and a universal exchange-correlation functional. A simplification is via pseudopotential theory (see Perdew paper in Ref. [1]). For each atom a so-called effective core potential or pseudopotential seen by the valence (chemically active) electrons is introduced. A very simple but effective one is the Ashcroft pseudopotential, which has applications to simple metals (see e.g. [3-5]).

The idea of a pseudopotential is to treat the valence electrons explicitly by DFT as interacting particles in the field created by the ions. The Hohenberg-Kohn variational equations for the real electronic system and in the pseudopotential method have to be identical. This condition gives the pseudopotential and the Kohn-Sham potential, $V_{\mathrm{KS}}(r, \sigma)$, for the system of the valence electrons. An ion acts on the valence electrons via the electrostatic potentials, a short-range repulsive component, $V^{\mathrm{R}}(r, \sigma)$, and the exchange-correlation interaction. In the local spin density approximations of the kinetic energy the repulsive potential has the following form: 


$$
V^{\mathrm{R}}(r, \sigma) \approx \frac{1}{2}\left\{6 \pi^{2}\left[n_{c}(r, \sigma)+n_{\mathrm{v}}(r, \sigma)\right]\right\}^{2 / 3}-\frac{1}{2}\left[6 \pi^{2} n_{\mathrm{v}}(r, \sigma)\right]^{2 / 3},
$$

where $n_{\mathrm{c}}(\boldsymbol{r}, \sigma)$ and $n_{\mathrm{v}}(\boldsymbol{r}, \sigma)$ are the spin densities of the core and valence electrons, respectively. In the following, all equations are expressed in the atomic units ( $\hbar=1$ a.u., electron mass $=1$ a.u., electron charge $=1$ a.u., the unit length is 1 bohr and energy unit is 1 hartree $=2$ Ry). In the calculations, the core orbitals are approximated by asymptotic expressions [6]. Exact calculations of close shells require the configuration interaction method. For valence electrons, the Kohn-Sham potential has the following form:

$V_{\mathrm{KS}}(r, \sigma)=\sum_{\alpha}\left[v\left(r-\boldsymbol{R}_{\alpha}\right)+V^{\mathrm{R}}\left(r-\boldsymbol{R}_{\alpha}, \sigma\right)\right]+\int \mathrm{d} r^{\prime} \frac{\varrho_{\mathrm{v}}\left(\boldsymbol{r}^{\prime}\right)}{\left|r-r^{\prime}\right|}+\frac{\delta E_{\mathrm{xc}}[\varrho, m]}{\delta n_{\mathrm{v}}(r, \sigma)}$.

Here, $v\left(r-\boldsymbol{R}_{\alpha}\right)$ is the electrostatic potential of the ion for the position $\boldsymbol{R}_{\alpha}$ of its nucleus. The term $v\left(r-\boldsymbol{R}_{\alpha}\right)+V^{\mathrm{R}}\left(\boldsymbol{r}-\boldsymbol{R}_{\alpha}, \sigma\right)$ in (2) is the pseudopotential of the core ion. The next term concerns the valence electrons (or itinerant electrons in metals) and is the Hartree (or direct, or classical) Coulomb potential. The exchange-correlation functional, $E_{\mathrm{xc}}[\varrho, m]$, is for the total ground state density: $\varrho(r)=n(\boldsymbol{r}, \uparrow)+n(\boldsymbol{r}, \downarrow)$, and the spin polarization $m(\boldsymbol{r})=n(\boldsymbol{r}, \uparrow)-n(\boldsymbol{r}, \downarrow)$, where $n(r, \sigma)=n_{\mathrm{c}}(r, \sigma)+n_{\mathrm{v}}(r, \sigma)$.

In our calculations of the spontaneous magnetization, we take into consideration the Dirac density matrix of the valence electrons (one-electron propagator). The density matrix is calculated iteratively. In the first step of the calculations, the density matrix is approximated by a uniform spin-polarized gas. In the next step, we apply the Lindhard approximation. In this approximation, the pseudopotential is determined for the spin densities of the previous calculations. The calculated spin moments (time-averages) per atom in this work for the uniform spin densities and in the Lindhard approximation are compared with experimental data in Table I. The magnetization is represented by a non-zero moment of the core ion due to the stability of the closed shells and the spin polarization of the valence

\section{TABLE I}

Calculated spin moments per Wigner-Seitz cell (in the atomic units): $S_{\text {uni }}$ and $S_{\text {Lin }}$ are for uniform valence spin gas and in the Lindhard approximation, respectively. Experimental moments [9] are denoted by $S_{\text {expt }}$.

\begin{tabular}{c|c|c|c}
\hline \hline $3 d$ metal & $2 S_{\text {uni }}$ & $2 S_{\text {Lin }}$ & $2 S_{\text {expt }}$ \\
\hline $\mathrm{Fe}$ & 2.0 & 2.5 & 2.22 \\
$\mathrm{Co}$ & 1.8 & 1.6 & 1.68 \\
$\mathrm{Ni}$ & 0.97 & 0.9 & 0.60
\end{tabular}


(chemically active) electrons. The magnetization of $\mathrm{Fe}$ or $\mathrm{Co}$ is determined by the moment of the $3 d^{5}$ closed shell and the spin polarization of the valence electrons. For nickel, we take two valence electrons. A cubic field splits the atomic levels of the $3 d$ shell into $E_{g}$ and $T_{2 g}$ subshells. In the nickel core, we take the $3 d^{5}$ spin up shell as well the $3 d^{3}, T_{2 g}$ spin down subshell. The ground-state properties of the $3 d$ transition metals have been studied by accurate density functional calculations in Ref. [7]; in particular, the magnetization values obtained there are in better agreement with experiment than the present ones (given in Table I). Our approach to the uniform electron gas is equivalent to the structureless pseudopotential approximation applied by Perdew et al. [5] to calculate the cohesive and surface properties of metals.

\section{The Bloch wall parameter in a molecular field approximation}

The conventional calculations of the magnon stiffness constant are related to the transverse dynamical spin susceptibility. Our calculations of the Bloch wall parameter, $A$, or the magnon stiffness constant, $D$, are based on a study of ferromagnetic and paramagnetic phases. In the statistical theory of order-disorder transitions, the Bragg-Williams approximation converts the energy functional of spin polarization into a simple sum of one-atom contributions

$$
E\left[\rho_{\mathrm{F}}, m_{\mathrm{F}} \neq 0\right]-E\left[\rho_{\mathrm{P}}, m_{\mathrm{P}}=0\right]=-\frac{1}{2}(2 J z) S^{2} .
$$

The Bragg-Williams relation(3) is set up in Ref. [8], where $J$ is studied. In the ferromagnetic and paramagnetic phases, $E\left[\rho_{\mathrm{F}}, m_{\mathrm{F}}\right]$ and $E\left[\rho_{\mathrm{P}}, 0\right]$ are the energy functionals per atom, respectively, $\rho_{\mathrm{F}}(r, T)$ and $\rho_{\mathrm{P}}(r, T)$ - the electronic densities, $\boldsymbol{m}_{\mathrm{F}}(r, T)$ and $\boldsymbol{m}_{\mathrm{P}}(r, T)$ - the spin polarizations. $T$ denotes the temperature. The parameter $J$ describes a spin-dependent, non-local interaction and in the case of the Heisenberg model it represents the exchange interaction between nearest-neighbour spin pairs. In our paper, the parameter $J$ is determined by Eq. (3) and represents the interaction between spin moments of the nearest-neighbour Wigner-Seitz cells. The number of the nearest-neighbour Wigner-Seitz cells is denoted by $z$. The ferromagnetic order parameter, $S=S(T)$, is the time-average of the spin moment per Wigner-Seitz cell along the axis of quantization. In the Heisenberg model, the parameter $J$ is an important physical quantity for ferromagnetic materials and affects the dispersion relation of magnons, the Bloch wall, the temperature properties (spontaneous magnetization vs. $T$, specific heat, Curie transition temperature) and the dynamic transverse magnetic susceptibility. The magnon stiffness constant, or the Bloch wall parameter is proportional to $J$, see [9]. The calculated values for $A$ and $D$ are compared with experimental data [9] in Table II. For Fe, a contribution of the discrepancy between theory and experiment in the magnitude of $D$ or $A$ relies upon the nearest-neighbour approximation. Numerical calculations of the energy of spin polarization are not simple and require sufficiently accurate computations. Our quite realistic numerical values of such quantities as the Bloch wall parameter, or the magnon stiffness constant are based on literature values of the energy of spin polarization [7]. Let us mention that better agreement between theory and experiment than that achieved here was obtained for $\mathrm{Ni}$ by Wang and Callaway [10]; however, they dealt with neither Co nor Fe. DFT theories 
TABLE II

The Bloch wall parameter, $A_{\text {cal }}$, and the magnon stiffness constant, $D_{\text {cal }}$, are calculated in this work. The experimental quantities $D_{\text {expt }}$ and $A_{\text {expt }}$ are taken from Ref. [9]. Energy functionals per atom (in [Ry]): $E\left[\rho_{\mathrm{P}}, 0\right]$ and $E\left[\rho_{F}, m_{F}\right]$ in the paramagnetic and ferromagnetic phases, respectively, are taken from Ref. [7]. Here $V_{\mathrm{ws}}$ is the volume of the Wigner-Seitz cell. $D$ and $A V_{\text {ws }}$ are expressed in Ry bohr ${ }^{2}$.

\begin{tabular}{c|c|c|c|c|c|c}
\hline \hline metal & $E\left[\rho_{\mathrm{P}}, 0\right]$ & $E\left[\rho_{\mathrm{F}}, m_{\mathrm{F}}\right]$ & $D_{\text {cal }}$ & $D_{\text {expt }}$ & $A_{\text {cal }} V_{\text {ws }}$ & $A_{\text {expt }} V_{\text {ws }}$ \\
\hline $\mathrm{Fe}$ & -2522.812 & -2522.833 & 0.135 & 0.075 & 0.073 & 0.041 \\
$\mathrm{Co}$ & -2760.760 & -2760.768 & 0.074 & 0.090 & 0.031 & 0.034 \\
$\mathrm{Ni}$ & -3011.649 & -3011.655 & 0.145 & 0.105 & 0.020 & 0.014
\end{tabular}

of $D$ are presented in Refs. [10,11]. The Hubbard model [12] value of $D$ is tested in Ref. [13], where calculated and experimental values of $D$ are compared for Co. Our calculations, which are based on the Bragg-Williams approximation and DFT method, take into account an effect of a magnetic disorder on the Wigner-Seitz cell. This contribution is absent in the conventional calculations of the magnon stiffness constant.

\section{Acknowledgments}

I would like to acknowledge some discussion with J. Morkowski and A.R. Ferchmin. This work was supported by the Committee for Scientific Research grant No. 2P302 00507.

\section{References}

[1] Density Functional Theory, Eds. R.M. Dreizler, E.K.U. Gross, NATO ASI Series B, Vol. 337, Plenum, New York 1995.

[2] Á. Nagy, Phys. Rep. 298, 1 (1998).

[3] N.D. Lang, W. Kohn, Phys. Rev. B 1, 4555 (1970); ibid. 3, 1215 (1971).

[4] J.H. Rose, J.F. Dobson, Solid State Commun. 37, 91 (1981).

[5] J.P. Perdew, H.Q. Tran, E.D. Smith, Phys. Rev. B 42, 11627 (1990).

[6] C. Almbladh, U. von Barth, Phys. Rev. B 31, 3231 (1985).

[7] V.L. Moruzzi, J.F. Janak, A.R. Williams, Calculated Electronic Properties of Metals, Pergamon, New York 1978.

[8] W. Schmidt, submitted to J. Phys., Condens. Matter.

[9] F. Keffer, Spin Waves, in: Encyclopedia of Physics, Ed. S. Flügge, Vol. XVI-II/2, Springer-Verlag, Berlin 1966.

[10] C.S. Wang, J. Callaway, Solid State Commun. 20, 255 (1976).

[11] K.L. Liu, S.H. Vosko, J. Phys. F, Metal Phys. 8, 1539 (1978).

[12] D.M. Edwards, R.B. Muniz, J. Phys. F 15, 2339 (1985).

[13] X. Liu, M.M. Steiner, R. Sooryakumar, G.A. Prinz, R.F.C. Farrow, G. Harp, Phys. Rev. $B$ 53, 12166 (1996). 